\title{
Radiation injury from x-ray exposure during brachytherapy localization
}

\author{
B. R. Thomadsen, ${ }^{a}$ B. R. Paliwal, ${ }^{a)}$ D. G. Petereit, ${ }^{\text {b) }}$ and F. N. Ranalloc) \\ University of Wisconsin-Madison, Department of Medical Physics, 1530 Medical Science Center, Madison, \\ Wisconsin 53706
}

(Received 21 December 1999; accepted for publication 10 April 2000)

\begin{abstract}
Two patients developed skin ulcers secondary to high doses of diagnostic-energy x rays received during localization procedures as part of brachytherapy treatments. Both were morbidly obese and diabetic. The obesity led to the delivery of estimated skin doses of $83 \mathrm{~Gy}$ in one case and $29 \mathrm{~Gy}$ in the other in attempts to produce readable images on localization radiographs. This report discusses the factors leading to the injuries, the progression of the injuries over time, and the variables involved in the localization procedures with the aim of preventing future mishaps. The greatest contribution to the large skin dose was the need, with the equipment available, to use multiple exposures to produce a single film, because of the effect of the resultant reciprocity failure.

(C) 2000 American Association of Physicists in Medicine. [S0094-2405(00)00807-5]
\end{abstract}

\section{INTRODUCTION}

Obesity is the primary risk factor for the development of endometrial cancer. These patients often have comorbid medical problems, such as hypertension, diabetes, and limited cardiopulmonary function. These same risk factors and comorbid medical problems can preclude surgical intervention (total abdominal hysterectomy and bilateral salpingooopherectomy) in 5\% or less of patients. Treatment alternatives include hormonal therapy, which is cytostatic, or radiotherapy, which is cytotoxic and curative in $85 \%$ of clinical stage 1 patients. Radiotherapy complications secondary to the brachytherapy procedure or the radiation dose have been reported.

After a long series of obese uterine cancer patients treated with high dose-rate brachytherapy (HDRB) without incident, little thought was given to the potential for injury to brachytherapy patients from the localization procedures. In general, the dose from the diagnostic radiographs given to radiotherapy patients pales by comparison to the therapeutic doses. Through the circumstances discussed below, two patients suffered from nonhealing skin ulcers secondary to the doses received during localization procedures using diagnostic radiographs.

\section{MATERIALS AND METHODS}

Two morbidly obese patients were treated for inoperable endometrial carcinoma using high dose rate brachytherapy. The normal localization procedure uses orthogonal radiographs. A ceiling-mounted $\mathrm{C}$-arm supports a diagnostic $\mathrm{x}$-ray unit and image intensifier permanently fixed horizontally for lateral imaging, with $74 \mathrm{~cm}$ between the bottom edge of the $\mathrm{x}$-ray tube collimator housing and the front of the intensifier. A second, permanently mounted $\mathrm{x}$-ray tube sits at the center of the $\mathrm{C}$-arm directed downward for anterioposterior (AP) radiographs. The receptor for the anterior beam slides under the patient in a cart on the floor, with a variable distance between the source and the patient. The imaging system used a 10:1 grid, DuPont Quanta III screens and Kodak XRP film. The generator and housing allowed a maximum of $110 \mathrm{kVp}$ and $500 \mathrm{mAs}$, and produced a $2.7 \mathrm{~mm} \mathrm{Al}$ half value layer at $80 \mathrm{kVp}$. The exposure rate for the lateral unit was $0.17 \mathrm{mGy} /$ $\mathrm{mAs}$ at $76 \mathrm{~cm}$ from the focal spot. The intersection of the AP and lateral beams (isocenter) falls $63 \mathrm{~cm}$ from the lateral focal spot and $59.5 \mathrm{~cm}$ from the AP.

Case 1: The first patient weighed $205 \mathrm{~kg}$ (450 pounds), stood $162 \mathrm{~cm}$ (5 foot 4 inches) and was diabetic. Other approaches for treatment were considered and dismissed. Her inability to lie in bed for more than a couple of hours without becoming hypoxic precluded low dose-rate brachytherapy (LDRB). Unlike LDRB using Heyman capsules with a treatment based on a time and strength product (mg Ra equivalent hours), HDR brachytherapy required calculation of dwell times for the actual geometry of the application.

A ceiling-mounted, C-arm radiography unit in the HDRB room was capable of making only orthogonal images with an anterior and a right lateral view (due to the orientation of utilities in the room such as oxygen lines). The danger of perforating her endometrium with the tandem precluded moving the patient for localization imaging to the simulator that had greater source to skin distances (SSD) available, or to a linear accelerator with a much higher energy x-ray beam.

This patient underwent the routine brachytherapy localization procedures using anterioposter (AP) and lateral radiographs except that imaging the laterals required multiple exposures on a single film. The $97 \mathrm{~cm}$ source to receptor distance allowed a $73 \mathrm{~cm}$ space between the collimator housing and the receptor for the patient, leaving $23 \mathrm{~cm}$ from the source to the collimator (SDD). The patient filled the available space, so the SSD became the $23 \mathrm{~cm}$ SDD. Initial radiographs using the maximum technique repeated three times produced no image. A second set of films taken using six exposures in a row also failed to produce an image. Twelve exposures for a single film finally produced a faint image, but one adequate for dosimetric calculations. The course of therapy included five treatments at weekly intervals, with dosimetry films taken at each fraction.

Performing 12 exposures to produce a single usable film results in a great loss of film sensitivity due to the phenom- 
TABLE I. Dose to the entrance skin due to imaging radiographs.

\begin{tabular}{lll}
\hline \hline \multicolumn{1}{c}{ Patient } & \multicolumn{1}{c}{1} & \multicolumn{1}{c}{2} \\
\hline Calibrated exposure rate & $0.17 \mathrm{mGy} / \mathrm{mAs} @ 76 \mathrm{~cm}$ & $0.17 \mathrm{mGy} / \mathrm{mAs} @ 76 \mathrm{~cm}$ \\
Inverse square correction & $(76 \mathrm{~cm} / 23 \mathrm{~cm})^{2}$ & $(76 \mathrm{~cm} / 23 \mathrm{~cm})^{2}$ \\
Exposure setting & $500 \mathrm{mAs} /$ exposure & $500 \mathrm{mAs} / \mathrm{exposure}$ \\
Exposures per film & 12 exposures $/$ film & 12 exposures $/$ film \\
Films for the Rx Course & $5.75 \mathrm{films}$ & $2 \mathrm{films}$ \\
Backscatter factor & 1.3 & 1.3 \\
Conversion & $1 \mathrm{~Gy} / 1000 \mathrm{mGy}$ & $1 \mathrm{~Gy} / 1000 \mathrm{mGy}$ \\
Total equivalent dose & $83 \mathrm{~Gy}$ & $29 \mathrm{~Gy}$ \\
\hline
\end{tabular}

enon of film reciprocity failure and therefore causes a large increase in patient dose. When using a screen-film cassette as the image receptor, the film is actually exposed by the light emitted by the intensifying screens in the cassette. The sensitivity of the film to this light diminishes greatly as the exposure time is increased beyond about $1 \mathrm{~s}$. This sensitivity loss is referred to as "reciprocity failure." With multiple exposures the total time over which the film is exposed to light may be many seconds to even minutes, and the reciprocity failure loss may be severe.

Table I details the calculation of the $83 \mathrm{~Gy}$ dose to the patient's skin from the localization procedures. The dose to the skin from the treatment with the ${ }^{192} \mathrm{Ir}$ source remained negligible.

Because of the dose produced by the multiple exposures, the patient developed a severe radiation dermatitis first noted after the last treatment fraction. The dermatitis involved a $9 \mathrm{~cm} \times 9 \mathrm{~cm}$ area corresponding to the entrance portal for the lateral localization $\mathrm{x}$ rays. The dermatitis progressed to an ulcer about $1 \mathrm{~cm}$ deep eight days later. After failing seven months of conservative treatment, the patient underwent debridment, a wide excision $(15 \mathrm{~cm} \times 10 \mathrm{~cm})$ and primary closure.

To prevent a similar occurrence in the future, the following changes in procedure were proposed:

(1) A change to faster screens and film (a gain of a factor of 3 in system speed).

(2) Replace the $50 \mathrm{~kW}$ tube with $100 \mathrm{~kW}$ tube and use 1000 $\mathrm{mAs}$ (a speed gain of a factor of 4 from the prevention of reciprocity failure loss based on measurement).

(3) A change from 10:1 linear grid to 6:1 cross grid. (This requires a slight increase in exposure of about a factor of 1.3 , but significantly improves the image contrast.)

(4) Rotate patient head to foot every other fraction to enter from opposite side.

According to measurements in the clinical situation, changes 1 and 2 together provide a gain of a factor of 4-5 in the system speed by allowing an image to be produced in a single exposure, minimizing speed loss due to reciprocity failure.

Case 2: The second patient weighed $200 \mathrm{~kg}$ (440 pounds) and was slightly wider than the first, and also diabetic. During localization procedures, it was discovered that, while the vendors had worked on the localization unit, none of the changes listed above had been made. For the first fraction, the patient received an initial 4-exposure radiograph, showing no image, followed by one using 12 exposures. At the patient's second insertion one week later, the patient's right side showed a mild erythema in the region of the lateral localization portal. To avoid further injury to the skin on the right side, the patient was rotated in the room so the localization beams entered the left lateral thigh using 12 exposures. The initial radiographs indicated that the applicator required adjustment. While a second set of radiographs taken following repositioning again indicated the need for further adjustment, the procedure was aborted due to patient discomfort and concern for the radiation exposure to the skin. For subsequent treatments, localization switched to a stereo-shift procedure using anterioposterior views, with related problems as discussed below. From the localization procedures this patient received 29 Gy to the skin on the left flank and 19 Gy on the right.

One week later at the third treatment session, the patient exhibited a marked erythema on the left side. Over 5 months, the erythema progressed from a dermatitis to a frank ulcer. The dermatitis on the right side cleared over period of three months. The injury on the left side persisted three years, spontaneously healing following conservative management. Figure 1 shows the skin during the erythema phase. The lead crosshairs provided enough protection to stand out as white lines against the reddened skin. Figure 2 shows the ulcer that developed.

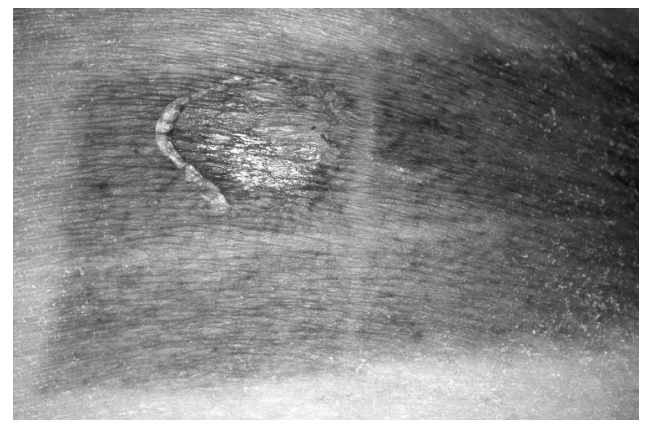

FIG. 1. Showing the radiation-induced ulceration in one of the patients. Notice the well-demarcated x-ray field, and the protection of skin shielded by the lead cross hairs. 


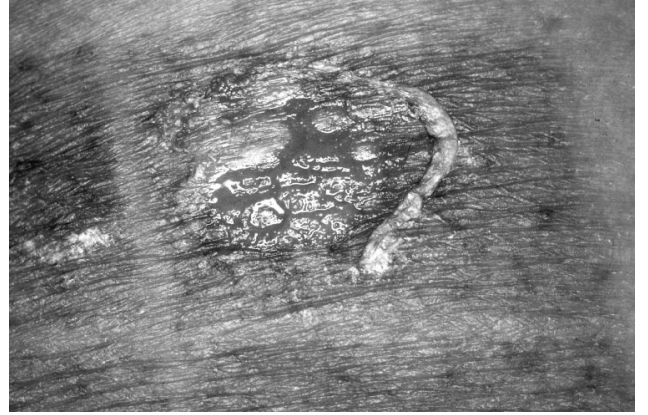

FIG. 2. Showing the progression of the injury into an ulcer.

\section{DISCUSSION}

The radiation oncology staff was surprised that this degree of skin injury could result from diagnostic x rays. Even though the localization procedures were supervised by a medical physicist certified in diagnostic medical physics, as well as radiotherapy physics, the level of hazard was not appreciated. Injury from high dose-rate fluoroscopy during long interventional procedures was known, but injuries resulting from obtaining readable radiographs were not. Typical exposures for lateral lumbar spine radiographs fall in the range of 5-15 mGy. ${ }^{1}$ Obviously, these were not typical patients. The calculated doses to the patients were verified through TLD measurements on phantoms simulating the patients' geometry, and read and calibrated by the University of Wisconsin Radiation Calibration Laboratory.

While the injury to the first patient followed from lack of attention to the radiation exposure levels at the patient's skin, the injury to the second patient resulted in part from failure to perform acceptance tests on the radiographic equipment after the assumed modifications.

Von Essen gives the single-fraction exposure corresponding to a $3 \%$ probability of skin necrosis as approximately 14.2 Gy (converted from his value of $1500 \mathrm{R}$ for a $100 \mathrm{~cm}^{2}$ field using orthovoltage $\mathrm{x}$ rays). ${ }^{2}$ Wagner et al. give the dose to produce erythema as approximately $6 \mathrm{~Gy}$, and $15-20 \mathrm{~Gy}$ for moist desquamation. ${ }^{3}$ It is interesting that the first patient, despite receiving a dose on the first fraction well exceeding the erythema dose, showed no signs of injury until after the full course of treatment, whereas the second patient developed an erythema 1 week after the initial exposure of 19.2 Gy.

Aggravating the problem of poor patient penetration with the diagnostic $x$ rays was the proximity of the patients' entrance skin to the x-ray unit's housing. The C-arm construction forced the patient into the $74 \mathrm{~cm}$ space between the receptor on the collimator housing, thus reducing the distance from the source to the skin, and markedly increasing the dose through the inverse-square relationship. Were ordinary radiographic equipment available, increasing the SSD, while requiring a higher total technique from the generator, would have reduced the relative dose to the skin for the required dose to the receptor.

The application of stereo-shift films posed multiple problems in this setting. The software for the HDR treatment- planning computer expected a shift between films across the patient. The patient couch construction allowed only a shift in the cephalocaudal direction. Bladder and rectal doses could not be calculated with the existing software using stereoshift reconstructions. The software expected both exposures on a single film, while, again because of table construction and image quality, each image was on a separate film. Because of lack of clearance between the patient and the $\mathrm{x}$-ray housing, shifting the unit also tended to move the patient. Resolving some of these problems merely extended the procedure time, while addressing others compromised the quality of treatment.

Conventional technology compromises treatment of such obese patients either by limiting the imaging possibilities, constraining the reconstruction options, or requiring moving of the patient (and the support team and equipment) risking injury and reducing the accuracy of treatment. Additional time spent grappling with the problems puts the patient in greater risk for phlebitis and pulmonary emboli. A faster screen/film system and a generator with twice the capacity could have reduced the exposure to the first patient by approximately a factor of 12 ; a factor of 3 due to the actual speed increase of the screen/film system, but also a factor of 4 from preventing speed loss from reciprocity failure. These changes would have reduced the first patient's skin dose to about 7 Gy for the course of treatment. Were a simulatortype radiography unit available in the procedure room, placing the film at $140 \mathrm{~cm}$ with the patient still in contact with the receptor would reduce the dose by another factor of 4-5 through the inverse square relationship, but would require a generator and tube capable of approximately $200 \mathrm{~kW}$. Increasing the filtration of the x-ray beam with $0.1 \mathrm{~mm}$ of copper reduces the entrance exposure to the patient by up to an additional factor of 2 without the need to adjust the technique, while producing images of equivalent quality. Quality assurance evaluation of the localization equipment on a semiannual basis forms an important tool to detect degradation in the imaging chain or changes in the unit that could increase the patients' skin dose. A diagnostic medical physicist, after evaluating the periodic quality assurance, might provide suggestions for incorporating new technology into the imaging process and reduce patient exposure.

Digital imaging, such as computed radiography (CR), might reduce the required doses and offer the possibility of enhancing the image to improve readability. CR is typically not as sensitive as general purpose screen/film systems, when exposed to provide equivalent image quality for short exposure times. However, CR does not suffer from the effects of reciprocity failure and may actually be significantly more sensitive if long exposure times are required. Also, by accepting an increase in image noise, CR and other digital imaging techniques may allow even further dose reductions. Unfortunately, the poor subject contrast may still dominate the resultant images.

Based on this experience, we recommend that all radiotherapy facilities assess the skin doses to patients undergoing localization radiography and determine whether there are 
limitations based on patient size for safely conducting the procedures.

\section{CONCLUSION}

Radiation oncology staff should be aware of the potential for severe complications in very obese patients due to doses from the localization radiographs, particularly for fractionated brachytherapy. Localization equipment, as with all equipment used in radiotherapy, requires careful analysis of the requirements of the image acquisition process when purchased. When received or modified, the equipment must be subject to a rigorous set of acceptance tests and a periodic quality control program. In particular, configuration of the imaging system requires care so that sufficient film density can be obtained in a single exposure to avoid the severe loss of system speed that results from reciprocity failure when films are created from multiple exposures.

${ }^{a}$ Departments of Medical Physics and Human Oncology.

${ }^{b}$ Department of Human Oncology.

${ }^{c}$ Department of Medical Physics and Radiology.

${ }^{1}$ P. Sprawls, Physical Principles of Medical Imaging (Medical Physics Publishing, Middleton, WI, 1993), p. 595.

${ }^{2}$ C. F. Von Essen, "A spatial model of time-dose-area relationships in radiation therapy,' 'Am. J. Roentgenol., Radium Ther. Nucl. Med. 81, 881-883 (1963).

${ }^{3}$ L. K. Wagner, P. J. Eifel, and R. A. Geise, "Potential biological effects following high $\mathrm{X}$-ray dose interventional procedures,' J. Vascular Interventional Radiol. 5, 71-84 (1994). 